\title{
Diferencias y semejanzas entre algunas exclamaciones en inglés medieval y en inglés moderno
}

\author{
Julia Fernández Cuesta
}

El propósito de este artículo es determinar qué tipo de exclamaciones aparecen más frecuentemente en la literatura de la época de Chaucer, para contrastarlas luego con las que aparecen en Shakespeare, cuyas obras son claramente representativas de lo que se denomina tradicionalmente Inglés Moderno. Para ello, hemos hecho una selección de algunas de las exclamaciones que aparecen con más frecuencia en obras de ambos periodos, y que podemos suponer, por tanto, que serían características del habla de cada uno de ellos.

Hemos comprobado que en Chaucer y otros autores medievales aparece un cierto tipo de exclamaciones que no encontramos más adelante en Inglés Moderno y, como contrapartida, la lengua de Shakespeare es rica en eufemismos que no existían en el periodo medieval. Este artículo pretende señalar algunos de los factores sociales que pudieron influir en la desaparición de unas exclamaciones y la aparición de otras en cada época histórica.

Para entender por qué se utilizaban un determinado tipo de exclamaciones en inglés medieval resulta indispensable tener en cuenta el transfondo socio-cultural de este periodo, es decir, conocer en qué tipo de sociedad vivían Chaucer, Gower, o el poeta de Piers Plowman, puesto que el habla de un pueblo está siempre sometida a la influencia de los acontecimientos históricos por lo que atraviesan sus gentes, por factores socioculturales y por el entorno social en general.

Lo primero que nos llama la atención cuando hojeamos la obra de cualquier autor medieval es el hecho de que la mayoría de las interjecciones que aparecen en ella son de carácter religioso. Sin embargo no encontramos exclamaciones de contenido sexual ${ }^{1}$.

\footnotetext{
${ }^{1}$ Hasta muy recientemente en la literatura no encontramos escritas exclamaciones de este tipo (cf. OED).
} 
No es sorprendente que la mayor parte de las exclamaciones sean blasfemias o invocaciones a Dios puesto que en la Edad Media lo religioso es el centro de la vida; EI juramento o la blasfemia, por lo que tiene de desafío y de ruptura de reglas, cobra evidentemente más sentido en una época imbuida de un profundo sentido transcendente de la vida que en nuestros días, en que lo humano, lo meramente existencial ha sustituido en muchos casos a lo religioso.

Las expresiones exclamativas de carácter religioso más corrientes en inglés medieval son las que hacen referencia a Dios, a la Virgen o a los santos puesto que todavía estamos en una Inglaterra anterior a la Reforma; también son muy frecuentes las que se refieren al diablo o a la condenación del alma, problemas todos que preocupaban a las gentes del medioevo.

La expresión más frecuente es din duda by God!, con sus múltiples variantes: by Goddes dignitee!, for Goddes Hert!, a Godes half!, for Godes sake!, etc. La palabra God' aparece, en ocasiones, modificada por un adjetivo: for verray God!, ye, holy God!, por un adverbio: by God above!, o por una oración de relativo: by God that this world made.

By God! puede tener diversa intensidad dependiendo del contexto. A veces equivale simplemente a indeed:
But this is yet the beste game of alle, That she for whom they han his this jolitee Kan hem therfore as muche thank as me. She woot namoore of al this hoote fare, By God, than a woot a cokkow or an hare. (CT, Kt, 1805-10)

y otras veces tiene mucha más fuerza expresiva, como en el caso siguiente:

'Nay' quod the Somonour, 'lat hym seye to me

What so hym list; whan it comth to my lot,

By God! I shal hym quiten every grot.

(CT, FrT., 1290-2)

Otro grupo de variantes de la expresión by God! son las del tipo: God help me so!, invocaciones a la divinidad, utilizadas muy frecuentemente por Chaucer, especialmente en boca de personajes femeninos:

As help me God, I laughe whan I thinke

How piteous a -nyght I made them swinke!.

(CT, WBT, 201-2) ${ }^{2}$

${ }^{2}$ En este caso (la comadre de Bath, burlándose de sus dos primeros maridos viejos) el sentido de la exclamación se podría interpretar en castellano como ¡Qué Dios me perdone!:

- ¿Qué dios me perdone por ponerme a reir cada vez que recuerdo cuán desesperadamente les hacía trabajar por las nochesj-. (trad. de Pedro Guardia para ed. Cátedra, p. 200). 
As help me verray God omnipotent ${ }^{3}$. (ibid., 423)

También tenemos documentada esta expresión exclamativa en Piers Plowman:

Of this matere I my th te namely well longe,

ac I shal seye as I sau ${ }^{\text {th }}$, so me god helpe.

(Passus V, 21-22)

También aparecen, en lugar de help, otros verbos como save, en expresiones como: «so god me save», blesse y spede, en: «so God me blesse» y «so God me spede». Algunas son más complejas y elaboradas como: «For so god wisly have mercy upon me» (FranT, 1475), «So wisely God my soule bringe in blisse» (MerT, 2171), «Bot vchon enlé we wolde were fyf-/ the mo the myryer, so God me blesse». (Pearl, 850).

Lord aparece también muy frecuentemente en la literatura medieval, y es una de las expresiones más corriente en Chaucer:

«But by that ilke Lord that for us bledde» (ShT, 178). Encontramos numerosos ejemplos de esta exclamación en The Miracle Plays; la mayoría de ellas tienen una función de súplica dirigida a Dios más que de juramento 'oath', como podemos comprobar en los siguientes ejemplos, en los que el personaje en cada caso se dirige a Dios con un claro vocativo:

Lorde! to be fede with the fode of thi fayre face. (York Plays, The Bakers, 76)

Lord! wyth a lastande luf we love the allone. (ibid., 57)

Encontramos también la exclamación Lord!, modificada por un adjetivo:

Gracyous Lord! Gracyous Lord! Gracyous Lord, como downe.

(Coventry Plays. The Salutation and Conception, 32)

$\mathrm{O}$ good Lorde! howe brode is my brest. (ibid., Thersites, 144)

Los juramentos por el nombre de Cristo aparecen en las obras medievales con más frecuencia que los que contienen el nombre de Jesús, que son solamente utilizados por los personajes más vulgares. Así en $C T$ jura por Cristo el carpintero: «by Jhesus, hevene Cyng». (MilT, 3464); los dos eruditos (clerks)en The Reeve's Tale: «For Christes peyne» (12), «For Cristes soule». (ibid., 4264). El cocinero jura por la Pasión de Cristo:

Ha! ha! quod he- for Cristes passion,

This millere hadde a sharp conclusion

Upon his argument of herbergage!. (CkT, 4325-7) ${ }^{4}$

También en Piers Plowman juran por Cristo personajes que pertenecen a las más diversas clases sociales, desde un rey:

${ }^{3}$ Con el mismo sentido que la anterior.

${ }^{4}$ En la versión de Cátedra ya citada se traduce - $\mathrm{J}_{\mathrm{i}} \mathrm{a}_{i} \mathrm{i} \mathrm{Ja}$ ! ipor la Pasión de Cristo! Los razonamientos sobre el hospedaje le han acarreado penosas consecuencias a este molinero. (159) 
By crist, quod the Kinge. (Passsus II, 192)

una mujer popular:

'By Crist' quod a comune womman thi companye will I folwe, ther shalt seye I am thi suster. I ne woot whwre thei bicome. (Passus V, 641-2)

y hasta la conciencia jura por Cristo:

Nay, by Criste' quod Conscience, congeye me (rather)!. (Passus IV, 4-5)

Jesús!, sin embargo, era un juramento más fuerte en la Edad Media, usado probablemente por el pueblo llano, y moralmente reprobable. Como en el caso de Christ!, hay que distinguir entre los simples juramentos y las invocaciones a la divinidad que contienen el nombre de Jesús. Entre estas últimas encontramos:

Jesu! on me thou have pittye, that I have moste in mynde.

(Piers Plowman, Passus IV, 415-16)

Our Lorde Jesus helpe me

For one letter here I can nat se (Everyman, 506-7)

En CT los juramentos por la Virgen están igualmente reservados a las clases más bajas. Algunos contienen solamente el nombre de María:

This wyf was nat afered not affrayed,

But boldely she seyde, and that anon:

«Marie, I deffie the false monk, daun Jon!. (ShT, 402) ${ }^{5}$

Otros juran ‘by Seynte Marie' (PardT, 685; MerT, 1899), y otras expresiones son más elaboradas:

Help, for hir love that is of hevene queene.

(MerT, 2334)

Son también frecuentes en este periodo los juramentos que hacen referencia a los santos, especialmente a San Juan y a San Jaime. Algunos de los santos invocados en la

\footnotetext{
${ }^{5}$ En este periodo todaviía encontramos el nombre de la Virgen en juramentos. En la literatura posterior será ya el eufemismo marry! el que aparecerá en lugar del nombre de María. En la edición de Robinson de $C T$ (1979) se dice que Marie! es equivalente a By St. Mary! (p. 962). Curiosamente, en la edición de Cátedra no se traduce esta exclamación:
}

Su esposa, ni asustada ni consternada, replicó seca y decididamente:

-Me importa un rábano ese monje embustero, el hermano Juan. (389) 
obras de Chaucer son, sin embargo, menos corriente. El carpintero de Oxford invoca a 'seinte Frydeswyde' (MilT, 3449), y en The Shipman' s Tale se pide ayuda a 'seint Denys of Fraunce' (ShT, 151). También encontramos logicamente expresiones que contienen el nombre de Santo Tomás Becket, puesto que es al santuario de Santo Tomás de Canterbury a dónde se dirigen los peregrinos; en algunas ocasiones aparece explícitamente el nombre del lugar junto al del santo: «By seint Thomas of Kent» (The House of Fame, 1131; The Miller's Tale, 3291). También se jura por santos locales, no tan conocidos como Santo Tomás. En The Miller's Tale aparece S. Neot en un juramento: «By seinte Note» (MilT, 3771) ${ }^{6}$. En CT se nos dice que el juramento más audaz de la priora era «by seinte Loy», lo que nos hace suponer que algunos juramentos por los santos eran exclamaciones de carácter muy suave:

Ther was also a Nonne, a PRIORESSE, That of hir smylyng was ful symple an coy; Hire gretteste ooth was but by Seinte Loy; And she was cleped madame Eglentyne. (GP, 119-21)

Otro santo que aparece en invocaciones o juramentos en $C T$ es 'seint Joce' (WBT, 481-84) ${ }^{7}$. También se jura por la cruz de Santa Elena. El anfitrión (host) se dirige al bulero (pardoner) airadamente al final del cuento:

Thoy woldest make me kisse thyn olde breech,

And swere it were a relyk of a seint,

Though it were with thy fundement depeint!

But, by the croys that Seint Eleyne fond,

I wolde I hadde thy coillons in myn hond

In stide of relikes or of seintuarie.

Lat kutte them off, I wol thee helpe hem carie

They shul be shryned in an hogges toord. (PardT, 950-5)

Thomas, en SumT jura por «seint Simon the Apostle»; el aguacil (the Summoner) invoca a la madre de la Virgen, Santa Ana, con un: «by the sweete seinte Anne», en FrT (1613).

${ }^{6} \mathrm{~S}$. Neot fue un santo sajón que vivió en el siglo XI.

${ }^{7}$ S. Joce fue un santo bretón del siglo VII, patrono de los peregrinos y cuya ermita llegó a ser el monasterio de St. Jessesu-mer, a media milla entre Abbeville y Calais.

${ }^{8}$ Nótese la "procacidad» del lenguaje en lo que se refiere a la terminología sexual. Sin embargo, como decíamos, no aparecen en los textos de la época palabras relacionadas con el sexo en expresiones exclamativas. La traducción de Cátedra es como sigue:

Vos me haríais besar vuestros calzones y juraríais que eran la reliquia de un santo, aunque los hubieseis ensuciado con vuestro culo. Por la Vera Cruz que encontró Santa Elena, antes agarraría vuestros cojones con la mano que vuestras reliquias y recuerdos. Desprendeos de ellos y os ayudaré a llevarlos y se los colocaremos en excrementos de cerdo. (378) 
En Piers Plowman se jura por San Pablo:

'Bi seint Poul!' quod the pardoner, parauenture I be noutht knowe there;/ I will go fecche my box with my breuettes and a bulle with bisshopes lettres. (Passus V, 63940)

En la poesía de Chaucer encontramos relativamente pocos juramentos por el diablo, y la mayoría son maldiciones. Aparece devil! con el mismo sentido que tiene en inglés actual. Algunas de las imprecaciones que contienen el nombre del diablo, reclamando la intervención de éste en asuntos humanos son bastante pintorescas:

The devil sette here soules bothe afyre! (LGW, 2439)

Hoold cloos thy mouth, man, by fader kyn!

The devil of helle sete his foot therein

Thy cursed breeth infecte wole us alle. (ManT, 37-8)9

«Hayt, Brok! hayt, Scot! what spare ye for the stones?

«The feend» quod he, yow fecche, body and bones,

As ferforthly as evere were ye foled,

So muche wo as I have with you tholed!

The devil have al, bothe hors and cart and hey!» (FrT, 1543-7)

Encontramos otros bastante representativos también en Troilus:

The devel spede hym that it recche. (Tr. IV, 630)

The foule feend me fecche. (Tr. I, 1610)

De todos los juramentos medievales los más censurados sin duda eran los que se referían a las partes del cuerpo de Cristo. Los predicadores de la época condenaron en sus escritos este tipo de juramentos. Wyclif escribió lo siguiente:

Also alle coyn swereris bi Goddis herte, bonys, nailis, and sidis, and othere membris, and fase and veyn swereris with lecchorours, and alle othere at comynly don athenst ony of Goddis hestis, for thei ben comyn mysdoeris, rennen, fully in this sentence ${ }^{10}$.

El propio Chaucer, por boca del cura 'parson' condena las blasfemias en las siguientes líneas:

${ }^{9}$ En el prólogo del cuento del intendente (Manciple), éste le reprocha al cocinero su embriaguez. La traducción de Cátedra es la siguiente:

No habráis la boca, hombre, por el amor de Cristo. ¡Qué el diablo de los infiernos meta el pie en ellaj Vuestro horrible aliento nos va a envenenar a todos. (530)

${ }^{10}$ John Wycliff, Select English Works of John Wycliff, (Oxford: Amold, 1871) vol. II, 332. 
For Cristes sake, ne swereth nat so synfully in dismembrynge of Crist by soule, herte, bones and body. For certe it semeth that ye thynke that the cursede Jewes ne dismembressed nat ynough the preciouse persone of Crist, but ye dismembre hym moore. (ParsT, 1537-41)

\section{Y también en PardT:}

Of sweryng seith the hooly Jeremye,

'Thou shalt swere sooth thyne othes, and nat lye

But ydel sweryng is a cursednesse.

Bihoold and se that in the firste table

Of heighe Goddes heestes honourable

Hou thet the seconde heeste of hym is this:

'Take nat my name in ydel or amys'.

Lo, rather he forbedeth swich sweryng

Than homycide or many a cursed thyngn;

'By Goddes precious herte', and 'By his nayles', And 'By the blood of Crist that is in Hayles,

Sevene is my chaunce, and thyn is cynk and treye!'

'By Goddes armes, if thou falsly pleye,

This daggere shal thurghout thyn herte go!'

Now, for the love of Crist, that for us dyde,

Lete youre othes, bothe grete and smale. (PardT, 635-660)

Sin embargo, en el mismo cuento, curiosamente, encontramos muchos juramentos de este tipo: «Goddes digne bones» (695), «Goddes armes» (692), etc. El habla del anfitrión (the host), de Harry Bailly, del aguacil (the summoner) y del bulero (the Pardonner) es rica en este tipo de exclamaciones; algunas de las más corrientes son for cokkes bones (ManT, 9; ParsT, 10), eufemismo de for Gods bones, o bi corpus bones (PhyT, 314; ShT, 1906). Otros como la Comadre de Bath (the Wife of Bath) o el fraile (the Friar) juran con bastante frecuencia, pero utilizan expresiones con menos alusión directa al nombre de Dios, y, en ese sentido, menos blasfemas. Lo mismo se puede decir de la expresión bi my fader soule, utilizada solamente por los personajes más rústicos de Chaucer.

Otra clase de juramentos que aparecen frecuentemente en la obra de Chaucer son: by my crown, by my thought, by my pan. Todos tienen un carácer coloquial, pero no están restringidos a un determinado grupo de hablantes.

\section{Exclamaciones de origen francés}

La influencia francesa en Inglaterra durante los siglos XII-XIII es de capital importancia en la historia de la lengua inglesa. Durante más de dos siglos el francés será la lengua de la corte, de la aristrocracia y de la administración. Aunque el inglés se sigue 
hablando durante todo el periodo, no cabe duda de la importancia y el prestigio superiores del francés. Así, cuando el inglés resurge como lengua «nacional» en el siglo XIII, después de la pérdida de Normandía, encontramos un lenguaje poblado de galicismos y de nuevos términos de origen francés. Los juramentos franceses se introducen también en la lengua y conviven con los antiguos anglosajones; como es lógico, las expresiones exclamativas de origen francés eran utilizadas por las clases más altas, mientras que el pueblo continuaba probablemente utilizando con preferencia las anglosajonas. Algunos ejemplos de interjecciones francesas que tienen su correspondiente en inglés son: par mafai, que corresponde a la inglesa upon my faith, opardee que equivale a by God. Otros juramentos que derivan directamente del francés son: parfay, pardieux, depardieux y mafay, entre otros. Parece ser que pardee era un juramento de carácter suave. En $C T$ lo utilizan el anfitrión (the host), el Caballero (the Knight), la Comadre de Bath (the Wife of Bath), y el Molinero (the Miller). Su origen era probablemente desconocido por muchos de los que lo usaban, y se utiliza en el mismo sentido que by God. La expresión mafai tenía un matiz cortesano. La utiliza el poeta de The Pearl:

Of countes, damysel, par ma fai

Wer fayr in heuen to holde asstate

Other elleth a lady of lasse aray ${ }^{11}$.

Encontramos frecuentemente en Chaucer la exclamación 'benedicite' (bless me), tomada del latín a través del francés en el siglo XIII:

What rowne ye with oure mayde? Benedicite!

Sire olde leccour, lat thy japes be!. (WBT, 241-2)

A juzgar por la evidencia de los textos, los juramentos por los dioses paganos que aparecen en la obra de Chaucer no eran frecuentes en el habla popular de la Edad Media entre el pueblo llano. Chaucer los utiliza, como en el caso siguiente tomado de Troilus and Criseyde, para reforzar el transfondo clásico de la obra:

And whan that Pandare herde hire name nevene, Lorde, he was glad, and sayde, «Friend so deere, Now far aright, for Joves name in hevene. (Tr. I, 878-80)

Por tanto, estas expresiones no son indicadoras de clase social sino que más bien añaden una connotación clásica a un cuento clásico. Por el contrario, los juramentos religiosos sí son, en Chaucer, indicadores de la clase social a la que pertenece el personaje.

Otros como by my trouthe hacen referencia a la fidelidad a la palabra dada, elemento de capital importancia en una sociedad en la que el amor cortesano tiene su más

${ }^{11}$ T. Silverstaein, ed. Medieval Lyrics. The Pearl (Oxford: Thomas Amold, 1971) 489-91. 
alta expresión en la literatura. Esta clase de expresiones aparecen en repetidas ocasiones en boca de Troilus:

Frend, as I am trewe Knyght

and by that feyth I shal to God and yow,

I hade it nevere half so hote as now

and ay the more that desir me biteth

To love hir best, the more it me deliteth. (Tr. III, 1648-52).

Con respecto a las interjecciones primarias que aparecen en las obras del periodo medieval, quizá una de las frecuentes sea $l o !$, procedente del anglosajón la!:

Lo, thon louely yle!. (The Pearl, 693)

Lo, Lorde, all readye heare $\mathrm{I}_{\mathrm{am}}{ }^{12}$

Woe! procede del anglosajón wa!, y aparece en inglés medio como expresión de dolor:

Alas, for woe!

Longer in land here I cannot abide!. (The sacrifice of Isaac, 253-4)

La interjección ho! procede del antiguo nórdico hó!, y no aparece documentada en anglosajón. Puede expresar placer, sorpresa, cansancio, y, en ocasiones tiene como finalidad llamar la atención de alguien: ho! there!, o what! ho!, etc. Como expresión de alegría la tenemos documentada en The Sacrifice of Isaac:

Ho! Isaake, Isaake, blessed muste thou be! (The sacrifice of Isaac, 325)

Como exclamación para llamar la atención:

What ho, fellows! Ho! Ho! Ho!. (Piers Plowman V, 64)

\section{Expresiones exclamativas en la época de Shakespeare}

Como decíamos al principio, nos ha parecido interesante tomar como referencia el teatro de Shakespeare para estudiar la diferencia entre las exclamaciones que aparecen en Chaucer y otros autores de la época, y las que encontramos a partir de Inglés Moderno. En la época isabelina seguimos encontrando exclamaciones de tipo religioso, si bien el contenido de éstas es ya diferente, principalmente a causa de la Reforma y del surgimiento del puritanismo en Inglaterra a partir del XVI. Es precisamente La Reforma, según Raleigh ${ }^{13}$, el único acontecimiento que viene a perturbar un panorama

${ }^{12}$ English Miracle Plays. Moralities and Interludes. Chester Plays. The Sacrifice of Isaac (Pollard) 210.

${ }^{13}$ W. Raleigh. The Age of Shakespeare. (Oxford: Oxford at the Clarendon Press, 1966), p. 54. 
que es, por otro lado, de gran seguridad y estabilidad política. Uno de los acontecimientos más significativos del siglo XVI es la crisis del feudalismo. Las ciudades, que ya han empezado a adquirir ciertos derechos, crecen rápidamente en detrimento de la vida del campo, de los grandes señorios feudales. El comercio se incrementa y surge una potente clase media que cada día se hace más poderosa. Esta clase media fomenta la aparición de las primeras 'Grammar Schools' con lo que la educación se extiende a un círculo mucho más amplio de la población y deja de ser patrimonio exclusivo del clero. Se hacen también frecuentes los viajes al extranjero, que complementan la educación del joven inglés, $\mathrm{y}$, como consecuencia de ello, se introducen en Inglaterra nuevas costumbres procedentes de los países que se visitan.

Parece que el estado de prosperidad de Inglaterra en este periodo es indiscutible; sin embargo, para algunos de los escritores de la época este renacimiento económico y cultural también tiene sus inconvenientes. Se atribuye a esta situación favorable y próspera la aparición de una relajación general de las costumbres. Muchos de los contemporáneos de Shakespeare no están muy satisfechos con los resultados de la creciente prosperidad de Inglaterra. En este sentido se pronuncia Stubbes (Anatomie of Abuses, 1583) cuando escribe:

For pride of mouth, heart and apparel, there is not a people ore abrupt, wicked and perverse upon the earth than the English.

Parece que sí existía una relajación general de las costumbres, e incluso una cierta peligrosidad social en la Inglaterra de Shakespeare. «The Holinshed Chronicle» (157787) nos cuenta como las peleas callejeras y riñas de tabema eran corrientes en este periodo, e incluso existía la costumbre de llevar armas:

Thou art like one of these fellows that when he enters the confines of a tavern, claps his sword upon the table, and says, God sends me no need of thee. (Rom., III, 1, 5-7)

Sin embargo, a pesar de todos estos inconvenientes, el nivel de vida era mucho más alto en esta época que en periodos anteriores; las casas eran más cómodas, y la alimentación mejoró, incluso entre las clases menos privilegiadas ${ }^{14}$.

También se introducen costumbres nuevas entre los ingleses, como la de beber cerveza. Parece que esta costumbre de bebery beber con exceso se extendió rápidamente entre la población. En 1607 el rey Jaime redactó un estatuto contra la embriaguez. En 1606 se había promulgado el «Decreto de Abusos» (Act of Abuses), que prohibía juramentos y blasfemias en el teatro. Podemos ver en estos dos estatutos promulgados con una diferencia de un año solamente el reflejo de ciertas costumbres consideradas decadentes y licenciosas por algunos coetáneos de Shakespeare. Elmismo Shakespeare, escritor nada sospechos de mojigatería, advierte de los peligros de la embriaguez en Hamlet:

${ }^{14}$ Cf. W. Raleigh The Age of Shakespeare. Oxford: Oxford at the Clarendon Press, 1966. 
The king doth wake tonight and take his rouse,

Keeps wassail, and the swaggering upspring reels,

And as he drains his draughts of Rhenish down

The kettledrum and trumpet thus bray out

The triumph of his pledge.

Hor. Is this a custom?

Ham. Ay, marry, it is.

But to my mind, though I am native here

And to the manner born, -it is a custom

More honoured in the breach than in the observance. (Ham. I-IV, 8-16)

Del mismo modo, esta Inglaterra más próspera y rica no carece de problemas sociales. Como consecuencia de los parcelamientos (field enclosures), grupos de campesinos y soldados sin trabajo se convierten en vagabundos que viven en muchas ocasiones del hurto y de la rapiña. Surge así la picaresca característica de la época isabelina.

Con la decadencia de la vida en el campo aumenta la población de la ciudades. Una de las mayores atracciones de la ciudad, dirigida a todas las clases sociales, es el teatro. Podemos considerar el teatro en el periodo isabelino, como The Canterbury Tales en la época medieval, una fuente de inestimable valor para el conocimiento del habla popular. Si bien, como decíamos al principio, la mayor parte de exclamaciones que encontramos en el teatro de esta época siguen siendo blasfemias o expresiones exclamativas de carácter religioso: Gods blood, Gods wounds, gods body, hay que señalar que, después del «Decreto de Abusos» (Act of Abuses), y como consecuencia del triunfo del puritanismo en Inglaterra, este tipo de juramentos va a desaparecer paulatinamente de la lengua, o al menos su uso se va a reducir notablemente. Por otro lado surgirán eufemismos que intentan disfrazar el nombre de Dios o de Cristo en expresiones como Cock, Gogs, Ods, etc. La mayoría de estas voces, que fueron muy populares en la época, han desaparecido hoy en la lengua.

La razón por la que la mayoría de estos juramentos tiene un carácter religioso (hasta el punto de que tiene que promulgarse una ley prohibiendo el uso del nombre de Dios, del Espíritu Santo y de la Trinidad en el teatro), es la misma que en la época de Chaucer: la supremacía de lo divino sobre lo humano, y la importancia de lo espiritual en la vida cotidiana, si bien en el periodo renacentista se pone ya en tela de juicio la idea de Dios como centro del universo. Un buen ejemplo es la figura de Hamlet, claro retrato de un humanista en una sociedad que todavía se apoya en valores medievales.

Con respecto a la ausencia de interjecciones de contenido sexual, el motivo es también el mismo que aducíamos anteriormente al referimos a la lengua de Chaucer; en tiempos de Shakespeare no existía ese pudor que en épocas posteriores convertirá en tabúes a este tipo de palabras. Los isabelinos no utilizaban eufemismo en materia sexual; por tanto el taco, la expresión soez como desafío, que tiene razón de ser en una sociedad donde todo lo relacionado con el sexo pertenece al ámbito privado de la vida y su tratamiento en el público está vedado, no ha lugar en este periodo en que las gentes se expresan abiertamente sobre estos temas: 
If my son were my husband, I would freelier rejoice in that absence wherein he won honour than in the ambracements of his bed where he should show more love. (Cor., Iiii, p. 34)

Hung him (poor cuckoldy knave) I know him not. I will use him as the key of the cuckoldy rogue's coffer, and there is my harvest-home». (Wiv., II iii, p. 60)

Estas expresiones y otras similares fueron la causa de que ciertos editores posteriores consideraran las obras de Shakespeare no aptas para leerse en el hogar y propusieran una edición expurgada de las mismas.

Quizá las exclamaciones más frecuentes en Shakespeare sean las maldiciones. Damned! ${ }^{15}$ y fie! ${ }^{16}$ aparecen en momentos de gran tensión dramática, cuando el personaje se encuentra bajo los efectos de una emoción fuerte. Otelo maldice a Desdémona cuando cree que ésta le es infiel:

O damn her, lewd minxi O damn her! damn heri. (Oth., IIIiii, 447)

Cuando se da cuenta de que le ha traicionado, Roderico maldice a Iago:

O damned Iago! O inhuman dog! (Oth., Viv. 63)

Damn con valor exclamativo tenía mucha mayor fuerza emocional en la época de Shakespeare que en la actualidad, y se utilizaba en situaciones bastante diferentes. En un autor moderno como Oscar Wilde, por ejemplo, damn! aparece en momentos de mucho menos tensión:

Damn, sir, it is your duty to get married. (An Ideal Husband, 208)

La diferencia es mucho mayor, desde el punto de vista semántico, si observamos que damn! se utiliza en la actualidad, en muchos casos, con un sentido positivo completamente desconocido en la época isabelina:

Damned good brandy!. (Priestley, The Linden Tree, 215)

${ }^{15}$ En el OED aparece la forma damned; como imprecación, (in imprecatory use) en el XVI. Acortada, como damn!', se encuentra a partir del XVII: «in clipped form damn from XVII»

${ }^{16}$ Fie! tiene una larga tradición lingüística. La primera documentación es de 1297. Sin embargo, el hablante actual la considera ya definitivamente una exclamación anticuada. Su origen es claramente onomatopéyico como se afirma en el OED: «An imitation of the sound instinctively made on perceiving a disagreeable smell». Este origen la une claramente a otras interjecciones de la lengua inglesa como fah!, que puede aparecertambién con las grafías faugh!, foh! , codificadas en los diccionarios, u otras más «vanguardistas», como Pf! o Ff!. El OED da como varientes gráficas $f i, f y$, fye, y phy.

Con respecto a la teoría sobre su descendencia del Antiguo Nórdico (Old Norse), cabe esta posibilidad, aunque también pudo surgir independientemente, es decir, puede tratarse de un desarrollo paralelo. De hecho, tenemos exclamaciones semejantes y con sentido parecidos en diversas lenguas, entre ellas las españolas iuf!, y ifu!. 
En alemán existe un uso similar de verdamnt, como modificador de un sustantivo en un sintagma nominal: «Verdamnt guter Mann» con el significado de: «very good man» ${ }^{17}$. thee»:

Fie! aparece seguida generalmente de un sintagma preposicional: «Fie upon

O, fie upon thee, slanderer. (Oth., IIi, 113)

$\mathrm{O}$, fie upon thee, strumpet. (Oth., VIv, 121)

En contextos semejantes se utiliza como exclamación la palabra death!, también seguida de un sintagma preposicional: «death on thy soul!». Macbeth maldice con esta expresión al joven soldado que le anuncia temeroso la llegada de las tropas inglesas:

Go prick thy face, and overred thy fear,

Thou lily-silvered boy

What soldiers patch?

Death on thy soul!

Those linen cheeks of thine

are counsellors to fear

What soldiers, whey-face?. (Mac. IIIiii, 398)

Las blasfemias, decíamos, son muy frecuentes en Shakespeare. Tenemos testimonios de que la misma reina Isabel era aficionada al uso de juramentos fuertes como God's Death $^{18}$. Sin embargo, ya en la época de Isabel I aparecen las primeras influencias del puritanismo que se reflejarán también en la lengua. Los puritanos, para quienes el centro de la vida lo constituía el trabajo y los servicios religiosos, atacaron sistemáticamente el teatro, considerándolo un lugar de corrupción.

El nombre de Dios aparece frecuentemente utilizado en Shakespeare hasta que el «Decreto de Abusos» prohibió su uso en el teatro; a partir de entonces aparece en la lengua un extenso repertorio de eufemismos. Pero hasta entonces lo encontramos a menudo como en el siguiente ejemplo de Hamlet:

O God, I could be bounded in a nutshell and count myself a king of infinite space, / were it not that

I have bad dreams. (IIii, 258)

${ }^{17}$ En el inglés coloquial es frecuente la expresión: «I know him damn well», en la que damn funciona como modificador de un adverbio.

${ }^{18} \mathrm{La}$ anécdota es como sigue: parece ser que un caballero de la orden de la Cruz Negra tenía órdenes muy precisas de no admitir a nadie a la presencia de la reina, y por lotanto prohibio el paso de un caballero, protegido de Leicester, que gozaba entonces del favor real. Como Leicester se lo reprochara duramente y se quejara a la reina, Isabel le respondio:

Gods death, my lord, I have whist thou well, but my favour is not so lockt up for you, that others shall not partake thereof. (W. Raleigh. The age of Shakespeare. Oxford: Oxford at the Clarendon Press, 1966, vol. 1, 5) 
For God! aparece también con bastante frecuencia, si bien se trata de un juramento mucho más suave y se emplea con mayor ligereza:

For God, an excellent song. (Oth., Iliii, 72)

La expresión Godes sollud no aparece en la edición del Folio. La mayor parte de las expresiones que se suprimen en la edición del Folio en esta obra aparecen en boca de Fuellen:

Godes sollud, if the enemy is an ass. (H5, IVi, 176)

Otros eufemismos que surgen ahora como consecuencia de esta nueva mentalidad son: gad!, cock!, gogs, (de God!), sblood! (for God's blood!), sdeath! (for God's death), etc., que se harán muy populares en el teatro del siglo XVII:

Upon the gad! Edmund, now, now! What news? (Lr. Iii, 21)

By Cock and pie, you shall not choose sir, come, come.

(Wiv. ITiii, 63)

Let him die. Sdeath thy impacience: throw cold water on my choler. (Wiv. IIIi, 64)

Swounds. Show me what to do. (Ham. Vi, 276)

Iago: Sblood, but yoy'll not hear me. (Oth. II, 4) Ii, 87ss.

Eufemismos de este tipo aparecen también documentados en Oth. IVi, 34ss, y Oth.

El nombre de Jesús aparecía en ciertas expresiones de las cuales la más corriente era 'by Iesus'. En ediciones posteriores de Shakespeare algunas expresiones que contenían el nombre de Jesús aparecen sustituidas por otras:

By Jesus (Q) there is very excellent services. (H5 IIIix, 62)

I assure you (F) there is very excellent services. (ibid., ed. Folio)

By Iesu(Q)

I say (F) I wil make him eat some part of my leek, or

I will play his pate four days» (H5 VIv, 38)

También encontramos eufemismos que intentan disfrazar el nombre de Jesús como es el caso de 'By Gis!':

By Gis \& by Saint Charity

Alack and fie for shame. (Ham. IVv, 58-9)

Una de las expresiones más frecuentes en las obras de Shakespeare es marry!, que procede del nombre de la Virgen. Parece ser que para los coetáneos de Shakespeare 
marry! no era más que un juramento suave «a linght oath», de cuyo origen, «By the Virgin Mary!», pocos eran probablemente conscientes.

La mujer jura tanto como el hombre en las obras de Shakespeare. Utiliza juramentos, e incluso juramentos fuertes:

Misstress Ford: Nay by the Mass that he did not. (Wiv. II, 94)

En esta época están todavía muy vivas entre las gentes las creencias en encantos, brujas y maleficios. Por tanto, encontramos en la lengua numerosas expresiones que hacen referencia al diablo; generalmente se trata de invocaciones a Satanás, o maldiciones en las que se pide al diablo que intervenga en contra de una determinada persona. En Macbeth se encuentra un ejemplo de este tipo de maldiciones:

The devil damn thee black,

Thou dream - fac'd loon!

where got'st thou that goose look? (Mac. Viii, 11-12)

y también en boca de mujeres como Bianca:

Let the devil and his dam

haunt you! What did you mean by

that same handkerchief you gave me even

now? I was a fine fool to take it. (Oth. IVi, 147)

También encontramos simples interjecciones que contienen el nombre del diablo:

Confess? Handkerchief? O devil!. (Oth. IVi, 43)

Darkness and devils!. (Lr. Iiv, 243)

Algunas expresiones exclamativas contienen la palabra 'soul'. Generalmente son juramentos por el alma del que jura. Así, Emilia jura por su alma cuando descubre el crimen de Othello:

You told a lie, an odious, damned lie,

Upon my soul, a lie, a wicked lie!. (Oth. Vii, 31)

Del mismo modo, cuando Desdémona es acusada de adulterio por su esposo, ésta jura por su alma para demostrar su inocencia:

Oth. That handkerchief which I so loved

and gave thee,

Thou gav'st to Cassio.

Des. No, by my life and soul. (Oth. Vii, 52)

Seguimos encontrando en Shakespeare exclamaciones de carácter más suave como heavens! (Ham. IIii, 134-5), heavenforbid! (Oth. Iiii, 254-3), o heaven havemercy 
on me! (Oth. Vii, 34ss) así como también la exclamación faith! (Lr. liv, 142). Estas exclamaciones se utilizan en momentos de menor tensión emocional, en situaciones de menor patetismo. Hamlet acude a una de estas expresiones (heavens!) cuando se le reprocha la tristeza que demuestra por la muerte de su madre, a pesar de que ha transcurrido ya algún tiempo desde el suceso:

\author{
O heavens! Die two \\ months ago and not forgotten yet? \\ Then there is hope a great's man memory \\ may outlive his life half a year. (Ham. IIii, 134-35)
}

Con respecto a las interjecciones primarias encontramos en Shakespeare algunas recurrentemente como holla! ${ }^{19}$, ha!, que ya aparecía en inglés medieval con diversos significados desde sorpresa hasta sarcasmo, y que ha sobrevivido en la lengua hasta la actualidad, a lo!, del anglosajón la. Ho! no aparece documentada en anglosajón, pero la tenemos documentada en la lengua desde el siglo XIII (cfr. OED). En Shakespeare es muy frecuente:

Help ho! They murder Caesar. (JC IIIii, 5)

Peace ho! Hear Antony (JC IIIii, 236-7)

R. What, ho, Brabantio! Signior Brabantio, ho!

I. Awake! Whant, ho, Brabantio! Thieves. (Oth. Ii, 78ss)

Como conclusión, podemos afirmar que en el inglés medio existía la costumbre de jurarmucho y de utilizar además expresiones y juramentos fuertes. Las exclamaciones utilizadas por todas las clases sociales, como se ha visto, son las que hacen referencia a Dios, a la Virgen y a los santos, esto es, las de contenido religioso. Esto es lógico si tenemos en cuenta que en la Edad Media lo espiritual tiene una importancia fabulosa en la concepción que el ser humano tiene del mundo. Como contrapartida, podemos afirmar que las interjecciones de carácter sexual no aparecen en los textos, a pesar (y quizá precisamente por ello) de la libertad con que se expresan los personajes de las obras medievales para referirse a todo lo que tenga que ver con el sexo, especialmente si comparamos las obras de esta época con las de etapas posteriores.

Evidentemente, no todas las clases sociales juran con la misma frecuencia ni tampoco utilizan el mismo tipo de juramentos. Las expresiones más vulgares aparecen en boca de miembros de las clases sociales menos privilegiadas, mientras que la aristocracia y el clero juran sólo en determinadas ocasiones, y utilizan expresiones mucho más suaves.

Por otro lado, se jura por motivos diferentes. En $C T$, una de las obras que mejor refleja los diferentes estratos sociales de la Edad Media, vemos como algunos de los personajes juran por costumbre, otros como reacción ante alguna emoción fuerte: miedo, sorpresa, dolor, etc., y otros para confirmar una promesa o defender su honor. Algunos no juran nunca.

${ }^{19}$ Del francés holà, aparece en inglés desde el siglo XVI (cfr. OED). 
La diferencia más significativa que encontramos en el uso de expresiones exclamativas entre la época de Chaucer y la de Shakespeare es la entrada en la lengua en esta última época (Inglés Moderno) de una serie de eufemismos para evitar pronunciar y el nombre de Dios y de la Virgen en contextos no sacros. A partir del «Decreto de Abusos» (Act of Abuses) (1606), muchas exclamaciones de carácter blasfemo desparecerán, y otras serán alteradas o sustituidas por eufemismos. Precisamente por esta razón encontramos las exclamaciones de las obras de Shakespeare sorprendentemente suaves comparadas con las que aparecen en Chaucer y en otros autores del periodo medieval.

Por los demás, las exclamaciones suelen ser de carácter religioso en su mayoría, semejantes a las que encontramos en la literatura medieval excepto las que contenían nombres de santos, que ya no serán tan frecuentes y desaparecerán progresivamente puesto que Inglaterra deja de ser un país «oficialmente católico» después de la Reforma. Por último, cabe destacar, como ocurría también en las obras medievales, la ausencia de exclamcaciones relacionadas con aspectos de la vida sexual debido a la libertad que todavía existe en el uso del lenguaje en este sentido.

\section{Bibliografía:}

Bühler, A. Vida y cultura de la Edad Media. Mexico: Fondo de Cultura Económica, 1956.

Chaucer, G. The Works of G. Chaucer. Ed. F. N. Robinson. 2nd. ed. Boston: Houghton, 1957.

Gordon, E. V. ed., Pearl. Oxford: Oxford at the Clarendon Press, 1980.

Ford, B. The Age of Shakespeare. Harmondsworth: Penguin Books, 1971.

Franklin, A. ed., Seven Miracle Plays. Oxford: Oxford UP, 1963.

Piers Plowman. The B Versión. Ed. G. Kane and E. Talbot Donaldson. London: The Athlone Press, 1975.

Pirenne, H. Las ciudades en la Edad Media. Madrid: Alianza Editorial, 1983.

Pollard, A. W. ed. English Miracle Plays, Moralities and Interludes. 8th ed. Oxford: Oxford at the Clarendon Press, 1961.

Romero, J. R. La Edad Media. Mexico: Fondo de Cultura Económica, 1956.

Shakespeare, W. Henry V. Harmondsworth: Penguin Books, 1979.

Shakespeare, W. Hamlet. The Signet Classic Shakespeare, Sylvan Barnet, 1963.

Shakespeare, W. Othello. Cambridge: Cambridge: Cambridge UP, 1979.

Shakespeare, W. Julius Caesar. Hardmondsworth: Penguin Books. 1980.

Shakespeare, W. Macbeth. Oxford: Oxford OP, 1965.

Shakespeare, W. King Lear. London: Penguin Books, 1958.

Shakespeare, W. The Merry Wives of Windsor. Harmondsworth: Penguin Books, 1957.

Shakespeare, W. Coriolanus. London: Penguin Books, 1955.

Shakespeare, W. The Merchant of Venice. London: Penguin Books, 1956.

Silverstain, T., ed. Medieval English Lirycs. London: Edward Amold LTD., 1971. 
Abreviaturas:

$\begin{array}{ll}\text { CT } & \text { Canterbury Tales } \\ \text { FranT } & \text { The Franklin's Tale } \\ \text { FrT } & \text { The Friar's Tale } \\ \text { GP } & \text { The General Prologue } \\ \text { Ham. } & \text { Hamlet } \\ \text { JC } & \text { Julius Caesar } \\ \text { KnT } & \text { The Knight's Tale } \\ \text { Lr. } & \text { King Lear } \\ \text { Mac. } & \text { Macbeth } \\ \text { MerT } & \text { The Merchant's Tale } \\ \text { MilT } & \text { The Miller's Tale } \\ \text { Oth. } & \text { Othello } \\ \text { PardT } & \text { The Pardoner's Tale } \\ \text { ParsT } & \text { The Parson's Tale } \\ \text { Rom. } & \text { Romeo and Juliet } \\ \text { SqT } & \text { The Squire's Tale } \\ \text { Tr. } & \text { Troilus and Criseyde } \\ \text { WBT } & \text { The Wife of Bath's Tale } \\ \text { Wiv. } & \text { The Merry Wives of Windsor }\end{array}$

